\title{
Inclusão social de pessoas com deficiência em Cabo Verde - África ${ }^{1}$
}

\author{
Maria Nivalda de Carvalho-Freitas \\ Nadine Helene Silva Rocha \\ Raquel Margarida Nascimento Lopes \\ Sónia Helena Lima Silva \\ Walter Leany Baptista Spínola
}

\begin{abstract}
RESUMO
O presente estudo investiga a percepção das dificuldades e possibilidades de inclusão social de Pessoas com Deficiência em três das dez ilhas do arquipélago de Cabo Verde - África. Em termos teóricos, utilizou-se o conceito de princípios de diferenciação de Bourdieu e de estigma de Goffman para a construção do instrumento de investigação. Foi aplicado um questionário a 67 PPDs nas ilhas de Cabo Verde. Em Cabo Verde, três fatores foram encontrados: percepção das relações sociais e aprendizagem; auto-estima e barreiras à cidadania. Constatou-se que, com o aumento da renda familiar, aumenta o nível de instrução das PPDs; que, quanto maior o nível de escolaridade, maior a renda familiar e menores são as dificuldades em relação às barreiras ao exercício da cidadania.
\end{abstract}

Palavras-chave: Cabo Verde, inclusão social, pessoas com deficiência.

\section{Título em inglês: SOCIAL INCLUSION OF PEOPLE WITH DISABILITY IN CAPE VERDE - AFRICA}

\begin{abstract}
The present study investigates the perception of difficulties and possibilities of social inclusion of People with Disabilities in three of the ten islands of the archipelago of Cape Verde. It was used both concepts the differentiation principles of Bourdieu and the stigma of Goffman to build the instrument of investigation. A questionnaire was applied in 67 People with Disabilities in Cape Verdean Islands. Three factors were considered: perception of the social relations; self-esteem and barriers to citizenship. It was verified that with the increase of the family income, the instruction level of People with Disabilities increases and smaller are the difficulties regarding the barriers to citizenship.
\end{abstract}

Index Terms: Cape, people with disability; social inclusion.

\footnotetext{
${ }^{1}$ Versão modificada do artigo apresentado e publicado no XXIX ENCONTRO ANUAL DA ASSOCIAÇÃO NACIONAL DE PÓSGRADUAÇÃO E PESQUISA EM ADMINISTRAÇÃO, 2005, Brasília. Anais eletrônicos. Brasília: EnANPAD, 2005. CD-ROM.
} 


\section{Introdução}

As pesquisas sobre a inserção de Pessoas Portadoras de Deficiência (PPDs) têm sido desenvolvidas principalmente nos continentes americano e europeu, e poucas pesquisas têm sido realizadas no continente africano. Nos últimos anos, tem havido um esforço mundial para o combate à fome e à pobreza, marcadamente nos países africanos, muitos deles assolados por conflitos étnicos, políticos e econômicos intermináveis. Em 1996, foi criada a Comunidade dos Países de Língua Portuguesa - CPLP - como um foro privilegiado de cooperação entre Angola, Brasil, Cabo Verde, Guiné-Bissau, Moçambique, Portugal, São Tomé e Príncipe e Timor-Leste. Além disso, o governo brasileiro promove o Programa de Estudante - Convênio de Graduação - PEC-G -, que possibilita a cidadãos da América Latina, do Caribe e da África cursarem a graduação, gratuitamente, no Brasil. A presente pesquisa foi viabilizada graças ao PEC-G, que facilitou a inclusão de alunos de Cabo Verde em projeto de pesquisa, o qual tinha por tema a inserção de PPDs no mercado formal de trabalho, bem como possibilitou a coleta de dados no país de origem dos alunos.

Cabo Verde é um país africano que foi colonizado por Portugal até 1975 , sendo a língua oficial o português e a língua materna o crioulo. Até 1990, Cabo Verde foi dirigido pelo partido que liderou a luta pela sua libertação. Atualmente, tenta consolidar o regime democrático, através de uma república parlamentar, tendo realizado aberturas política e econômica, além de buscar no capital estrangeiro um aliado para seu desenvolvimento. É um país de clima desértico com carência de recursos naturais, sendo muito dependente de importações. Segundo dados do Ministério das Relações Exteriores do Brasil (2005), mais de $50 \%$ da população caboverdiana emigra para os países da Europa e para o continente americano em busca de melhores condições de vida; contudo, continuam encaminhando seus recursos para o país de origem. O turismo é uma das principais apostas de investimento nele realizadas, devido à beleza e à diversidade das dez ilhas que o compõem. Segundo dados do Censo 2000, a população é de aproximadamente 450.000 habitantes, a maioria de origem africana; a população de PPDs é de 13.948 habitantes, o que representa $3,2 \%$ de pessoas com pelo menos alguma incapacidade de andar, ouvir ou enxergar; deficientes mentais; paraplégicos; falta de membro ou parte dele (INSTITUTO NACIONAL DE ESTATÍSTICA, 2005). Não existem, no país, leis que obriguem a contratação de PPDs por parte das empresas ou entidades públicas, apenas incentivos às 
empresas, através da redução nos encargos relativos a impostos sobre o rendimento (CABO VERDE, 2001).

No mundo, de uma forma geral, a questão da inserção de PPDs nunca foi uma preocupação mais sistematizada do mercado de trabalho. Somente após a Segunda Guerra Mundial, o direito ao trabalho da PPD foi legalizado, em função dos ex-combatentes que voltavam mutilados da guerra, sendo esse direito estendido às demais PPDs. No século $\mathrm{XX}$, foram criadas, no mundo inteiro, instituições especializadas, as quais passaram a apoiar a equiparação de oportunidades para as PPDs e a criar um intercâmbio de conhecimentos sobre a deficiência.

Segundo Goss, Goss e Adam-Smith (2000), desde a década de 1940, a Europa tem adotado o esquema de cotas de emprego para ex-combatentes, aplicável a outras PPDs, como uma forma de caridade social. Segundo esses autores, sob essa visão de caridade estão duas poderosas ideologias: o "modelo médico" e o "modelo da tragédia", ambos dando forte ênfase à perspectiva "profissional" e à legislação para a comunidade. O "modelo médico" define a deficiência como um atributo individual, resultado de uma patologia, e que necessita de uma intervenção profissional para sua "retificação". O "modelo da tragédia", baseado no senso comum, é complementar ao "modelo médico" e defende a existência de instituições que cuidem das PPDs e incentivem a difusão de donativos, como uma forma de caridade para essas instituições. Ambas perspectivas sobre as PPDs estão calcadas na premissa de que elas não são capazes de cuidar de si mesmas ou de fazer escolhas para suas próprias vidas, enfatizando uma percepção negativa, se comparadas às pessoas não portadoras de deficiência.

Ainda segundo Goss, Goss e Adam-Smith (2000), a partir das décadas de 1960 e 1970, a dominância dos modelos ideológicos "médico" e "da tragédia" tem sido questionada pelos movimentos sociais, notadamente nos Estados Unidos, onde o movimento pelos direitos humanos e civis foi fortemente defendido. Esse questionamento, fruto de um movimento da sociedade, dos grupos minoritários e das próprias PPDs, contribuiu para o surgimento de uma nova perspectiva ideológica em relação às PPDs, o "modelo social", que possui forte ênfase nos direitos civis e na iniciativa individual. Segundo Batavia e Schriner (2001) o "The Americans with Disabilities Act", a regulamentação dos direitos das PPDs nos Estados Unidos, baseia-se nos direitos civis de grupos minoritários, no modelo de vida independente e busca a eliminação da discriminação das PPDs, colocando como centro de sua ação legal a eliminação das discriminações sociais e das desvantagens sentidas pelas PPDs. 
$\mathrm{Na}$ atualidade, os estudos sobre a inserção de PPDs se posicionam, de forma explícita ou implícita, sob a égide de dois paradigmas políticos internacionais dominantes sobre a deficiência. Um paradigma, derivado dos Estados Unidos e do Canadá, centra-se em fortes medidas antidiscriminação, com ênfase nos direitos civis e no modelo de vida independente por parte da PPD (BATAVIA; SCHRINER, 2001; GOSS, GOSS, ADAMSMITH, 2000). Outro, proveniente dos demais países europeus, depende, substancialmente, de cotas compulsórias de emprego e de uma extensiva ação do Estado (GOSS, GOSS, ADAM-SMITH, 2000). Cabo Verde tem-se alinhado aos países europeus, buscando a arbitragem do Estado para as questões da inserção de PPDs.

As pesquisas, tanto nacionais quanto internacionais, revelam as dificuldades que essas pessoas enfrentam para ingressarem, se manterem e crescerem dentro das empresas (LEWIS; ALLEE, 1992); a falta de preparo do mundo industrial para absorvê-las, devido às adaptações de postos de trabalho as quais precisariam ser feitas em alguns deles (SILVA, 1993); a necessidade de se repensar as práticas de Recrutamento e Seleção por parte das empresas (FREITAS; MARQUES; SCHERER, 2004); a maior chance de se contratar PPDs por parte de grandes empresas que tenham profissionais de Recursos Humanos (GOSS; GOSS; ADAM-SMITH, 2000); e as contradições por parte das empresas ao contratar PPDs, que vão desde a segregação, contratando-as por grupos e categorias, em trabalhos considerados inferiorizados, mantendo atitudes de discriminação, até atitudes de inserção que reconhecem o desempenho das PPDs e a necessidade de a empresa se adequar às necessidades diferenciadas das pessoas (BATISTA, 2004).

Por outro lado, pesquisas mostram que o trabalho, associado a um emprego (mercado formal), desempenha um papel importante para os grupos historicamente marginalizados, tais como as PPDs, diminuindo as taxas de pobreza, propiciando a diminuição do isolamento social, aumentando sua participação política (SCHUR, 2002); aumentando sua auto-estima (OLIVEIRA, 1993); fazendo-os sentirem-se cidadãos completos, independente de suas limitações físicas (MARTINS, 1996), e sendo uma dimensão interdependente da percepção que as PPDs têm de si mesmas e da própria vida (FREITAS; MARQUES; SCHERER, 2004).

A presente pesquisa teve como objetivo contribuir para ampliar o conhecimento sobre a realidade das PPDs, em Cabo Verde, através da verificação da percepção das PPDs quanto às suas possibilidades e dificuldades de inserção social e no mercado formal de trabalho. 
O texto está organizado em quatro partes, além dessa introdução: o referencial teórico adotado na pesquisa; a metodologia; a análise de dados e as considerações finais.

\section{Referencial teórico}

Para entender como ocorre a inserção das PPDs, é necessário partir do pressuposto de que elas são pessoas que se diferenciam da população em geral, quer seja pela incapacidade gerada pela deficiência, quer seja pela desvantagem social decorrente da ausência de condições que possibilitem a independência e a autonomia delas. Nesse sentido, é preciso verificar a questão da inserção dentro de uma sociedade pautada pelas diferenças.

Bourdieu (1996) criou uma nova construção teórica, também denominada como filosofia da ação, e alguns conceitos fundamentais - como habitus, campo e capital - os quais funcionam como princípios para uma leitura relacional e também geradora, isto é, capaz de ajudar os pesquisadores a definirem os princípios fundamentais de diferenciação, aqueles que tornam as diferenças significativas, distintivas, nos espaços sociais. Esse autor entende o espaço social ou campo como uma rede de relações objetivas entre posições. Cada posição só podendo ser definida por sua relação objetiva com outras posições, ou seja, só podendo apreender as características de uma posição através de sua relação com as demais posições daquele espaço social. Todas as posições dependem de sua situação atual e potencial na estrutura de distribuição de poder (tipo e volume de capital), cuja posse determina a obtenção de lucros específicos. O poder simbólico é a concretização do reconhecimento do valor de um capital específico que confere aos seus detentores o poder de fazer ver o que não é visto e de legitimar uma forma de compreender e ver o mundo (BOURDIEU, 1996, 1998).

Bourdieu e Wacquant (1992) afirmam que, apesar de os diferentes campos possuírem seus capitais específicos, as sociedades atuais têm nos capitais econômico, cultural e social seus mais importantes princípios de diferenciação. Por capital econômico entende-se a posse de rendas, patrimônio, bens materiais e recursos financeiros em geral. $\mathrm{O}$ capital cultural refere-se principalmente à educação legitimada pelas instituições escolares. O capital social refere-se à rede de relações que possui um indivíduo e que lhe dá possibilidades diferenciadas em seu espaço social. A concentração desses diversos tipos de capital transforma-se em poder simbólico e confere prestígio ao seu detentor. 
Além disso, é preciso considerar que as PPDs ocupam uma posição diferenciada na estrutura social a qual, conforme Goffman (1982, p. 137), é fruto da história e das estratégias desse grupo e, também, das "contingências que essas pessoas encontram na interação face-a-face". A posse de uma deficiência é entendida como um atributo depreciativo em relação à pessoa que o possui. Esse atributo recebe um valor negativo (estigma) no seio das relações sociais, pois "a sociedade estabelece os meios de categorizar as pessoas e o total de atributos considerados como comuns e naturais" (GOFFMAN, 1982, p. 11) para uma determinada categoria social.

Assim, o estigma é considerado um critério de diferenciação utilizado em relação às PPDs. Esse processo de estigmatização envolve um duplo movimento: uma atribuição, por parte da sociedade, de um valor depreciativo para as PPDs, e uma legitimação, maior ou menor, por parte dessas pessoas, das características que lhe são atribuídas. O resultado desse duplo movimento, de atribuição e de legitimação, orientará ou constrangerá as percepções que as PPDs têm a respeito de si mesmas, de sua aceitação social e de suas possibilidades.

Dessa forma, para analisar a questão da inserção social e no mercado de trabalho por parte das PPDs, é necessário contemplar as principais formas de diferenciação adotadas pela sociedade, sua abertura, maior ou menor, para as mudanças que impliquem uma adequação da sociedade para todos, e os valores internalizados pelas PPDs, isto é, as possibilidades, maiores ou menores, dessas pessoas superarem as dificuldades e restrições sociais.

A partir dessas perspectivas, a proposta da pesquisa é verificar qual é a percepção das PPDs de Cabo Verde, quanto às suas possibilidades de inserção social e no mercado formal de trabalho, levando-se em consideração o posicionamento dessas pessoas quanto à posse dos capitais econômico, cultural e social; e às dificuldades e facilidades que sentem em decorrência do estigma social relacionado à deficiência.

\section{Método de pesquisa utilizado}

A pesquisa foi desenvolvida em três etapas: contextualização histórica e cultural do país; revisão da literatura, aprofundamento do referencial teórico e adequação semântica dos instrumentos de pesquisa; e aplicação do questionário à amostra de PPDs.

Em todas as etapas da pesquisa a presença dos bolsistas, oriundos de Cabo Verde, foi fundamental para a consecução da investigação. 
O questionário utilizado foi uma adaptação, feita a partir de uma pesquisa realizada no Brasil (FREITAS; MARQUES, SCHERER, 2004). Contudo, precisou sofrer modificações, tais como redação de algumas questões, moeda do país para definição da renda familiar, e nomeação adequada dos níveis educacionais, para adaptar-se à realidade do país em que seria aplicado.

O questionário definitivo foi composto por questões fechadas, com a utilização da escala Likert para medição, considerando-se uma variação de 1 (discorda totalmente) a 5 (concorda totalmente). A opção pelo questionário foi devida à escolha prévia dos fatores discriminantes, realizada a partir do referencial teórico utilizado, levando-se em conta os princípios de diferenciação propostos por Bourdieu $(1996,1998)$ e os estigmas atribuídos e assumidos pelas PPDs, conforme Goffman (1982).

A pesquisa foi realizada no ano de 2004, em três das dez ilhas do país, a saber: Ilha do Sal, Ilha de São Vicente e Ilha de Santo Antão. Essas três ilhas possuem, conjuntamente, uma população de 121.962 habitantes, sendo 4.814 PPDs, o que representa 3,95\% de portadores de deficiência. Em função da dificuldade de ser composta uma amostra aleatória, optou-se por trabalhar com uma amostra de conveniência. $\mathrm{O}$ critério adotado foi a possibilidade de acesso às PPDs. Os questionários foram respondidos por 67 PPDs, com idade superior a 16 anos, as quais constituíram a amostra pesquisada.

Os dados coletados, através do questionário, foram analisados com o suporte do programa estatístico SPSS. Foram realizadas a análise descritiva dos dados e a análise fatorial, posto que se buscava identificar os fatores que explicassem a percepção das PPDs quanto às suas possibilidades e dificuldades de inserção social e no mercado formal de trabalho. O tratamento preliminar dos dados resultou na exclusão das questões para as quais foi identificada ocorrência de missing values superior a $30 \%$ do total de respostas. $\mathrm{O}$ índice de Kaiser-Meyer-Olkin $(\mathrm{KMO}=0,682)$ e o teste de esfericidade de Bartlett (significativo a $\mathrm{p}<0,003$ ) indicaram a fatorabilidade dos dados.

Além disso, procedeu-se à análise da precisão do instrumento de coleta de dados, através do método de consistência dos itens, considerando o coeficiente alfa de Cronback. Dos 4 fatores encontrados, 3 apresentaram consistência interna superior a 0,70, o que, de acordo com Malhotra (2001), pode indicar a confiabilidade satisfatória da consistência interna. Em relação ao quarto fator extraído, foi encontrado o coeficiente alfa de Cronbach de 0,45, o que motivou sua exclusão da análise.

Também foram verificadas as relações de variação entre os fatores e as variáveis demográficas - renda familiar e grau de escolaridade -, visando analisar a possível 
correlação entre esses dados, posto que o capital econômico e o cultural estão sendo considerados fatores discriminantes, a partir do referencial teórico adotado.

\section{Apresentação e análise dos resultados}

Primeiramente, será apresentada a caracterização da amostra pesquisada, com o auxílio da estatística descritiva; depois, serão descritos os fatores e sua interpretação, obtidos através da análise fatorial que visava à validação do instrumento de pesquisa proposto; e, por último, será apresentada a correlação entre os fatores e as variáveis demográficas de renda familiar e escolaridade investigadas. Além disso, serão discutidas as principais constatações verificadas nas entrevistas realizadas nas empresas e na associação de PPDs pesquisadas.

\section{a) Caracterização da amostra}

Para a caracterização da amostra serão apresentados dados de identificação e indicativos sócio-demográficos na Tabela 1.

\begin{tabular}{|c|c|c|c|}
\hline Variável & Resultados & Variável & Resultados \\
\hline Idade & Média $=35,35$ anos & $\mathrm{N}^{\mathrm{o}}$ filhos & Média $=1,12$ filhos \\
\hline Sexo & $\begin{array}{c}\text { Masculino }=56,7 \% \\
\text { Feminino }=43,3 \%\end{array}$ & Estado civil & $\begin{aligned} \text { Casado } & =13,4 \% \\
\text { Solteiro } & =85,1 \% \\
\text { Outros } & =1,5 \%\end{aligned}$ \\
\hline Renda familiar & $\begin{array}{c}\text { Até } 8 \text { mil escudos }=41,7 \% \\
\text { De } 8 \text { a } 10 \text { mil escudos }=30,0 \% \\
\text { De } 10 \text { a } 30 \text { mil escudos }=13,3 \% \\
\text { De } 30 \text { a } 50 \text { mil escudos }=3,3 \% \\
\text { Mais de } 50 \text { mil escudos }=11,7 \%\end{array}$ & $\begin{array}{c}\text { Nível de } \\
\text { Escolaridade }\end{array}$ & $\begin{array}{c}\text { Analfabeto }=23,9 \% \\
\text { Primário incompleto }=25,4 \% \\
\text { Primário completo }=10,4 \% \\
\text { Liceu incompleto }=17,9 \% \\
\text { Liceu completo }=7,5 \% \\
\text { Escola técnica }=4,5 \% \\
\text { Superior incompleto }=6,0 \% \\
\text { Superior completo }=4,5 \%\end{array}$ \\
\hline $\begin{array}{c}\text { Tipo de } \\
\text { deficiência }\end{array}$ & $\begin{array}{c}\text { Motora }=10,4 \% \\
\text { Mental }=21,0 \% \\
\text { Auditiva }=9,0 \% \\
\text { Visual }=9,0 \% \\
\text { Multideficiências }=10,4 \% \\
\text { Física }=40,3 \%\end{array}$ & $\begin{array}{l}\text { Atividade de } \\
\text { trabalho }\end{array}$ & $\begin{array}{c}\text { Não trabalha }=65,2 \% \\
\text { Atividade formal }=19,7 \% \\
\text { Atividade informal }=12,1 \% \\
\text { Atividade voluntária }=3,0 \%\end{array}$ \\
\hline
\end{tabular}

Tabela 1. Caracterização da Amostra Pesquisada ( $n=67$ PPDs). Fonte: Dados da pesquisa, 2004. 
Os dados apresentados demonstram que a idade média dos pesquisados era de 35 anos, com ligeira predominância de pessoas do sexo masculino. O estado civil predominante entre os pesquisados é solteiro, com $85,1 \%$ dos respondentes nessa categoria. Os sujeitos da pesquisa declararam, em sua maioria $(71,7 \%)$, renda familiar de até 10 mil escudos, o que equivale a aproximadamente $\mathrm{R} \$ 300,00$ (trezentos reais), no Brasil. O nível de escolaridade é predominantemente baixo, com 59,7\% das pessoas possuindo, no máximo, o primário completo ${ }^{2}$; contudo, PPDs possuidores de formação técnica $(4,5 \%)$ e de curso superior completo $(4,5 \%)$ fizeram parte da amostra. Sobre o tipo de deficiência apresentado pelos pesquisados, a deficiência física foi predominante, com 40,3\% dos respondentes, seguido pela deficiência mental $(21,0 \%)$. Finalmente, verificou-se que 65,2\% das PPDs que participaram da investigação não trabalhava. Das pessoas que trabalhavam, 19,7\% atuava no mercado formal; $12,1 \%$ realizava alguma atividade informal; e 3\% exercia atividade voluntária. Esse quadro de referência demonstra a caracterização dos sujeitos que foram pesquisados.

\section{b) Os fatores e sua interpretação}

A interpretação descrita a seguir está baseada nas definições teóricas e metodológicas adotadas.

\begin{tabular}{ccccc}
\hline Item & carga & $\mathbf{h}^{\mathbf{2}}$ & $\mathbf{x}$ & Descrição \\
\hline 01 & 0,76 & 0,79 & 3,91 & Ser considerado pela família como uma pessoa capaz de tomar decisões \\
02 & 0,40 & 0,16 & 2,53 & Não se sentir capaz de aprender quando estava na escola \\
03 & 0,39 & 0,41 & 2,45 & Não ser tratado com preconceito \\
\hline
\end{tabular}

Tabela 2. Matriz Fatorial do Fator Percepção das Relações Sociais e Aprendizagem (Alfa = 0,92). Fonte: Dados da pesquisa, 2004.

Esse fator representa o construto que expressa a percepção das PPDs sobre as relações sociais e aprendizagem. Verifica-se que a confiança depositada pela família na capacidade da PPD em tomar decisões está relacionada com a forma como a PPD percebe o preconceito social e com o sentimento de capacidade de aprendizagem na escola. Em relação à amostra pesquisada, as médias encontradas demonstram uma tendência à neutralidade em relação a essas questões, o que é explicado em função da característica da amostra, no que se refere à escolaridade.

\begin{tabular}{|c|c|c|c|c|}
\hline Item & carga & $\mathbf{h}^{2}$ & $\mathbf{x}$ & Descrição \\
\hline
\end{tabular}


$\begin{array}{lllll}04 & 0,88 & 0,70 & 3,25 & \text { Ser considerado inferior pelas pessoas não portadoras de deficiência }\end{array}$

$05 \quad 0,32 \quad 0,81 \quad 4,55 \quad$ Gostar de si mesmo

Tabela 3. Matriz fatorial do fator auto-estima $($ Alfa $=0,72)$. Fonte: Dados da pesquisa, 2004.

Esse fator indica que as pessoas associam a relação de igualdade percebida nas relações sociais com o gostar de si mesmas. Na amostra pesquisada, as pessoas afirmam gostar de si mesmas, contudo têm uma pequena tendência a concordarem que as pessoas as consideram inferiores, em relação às não portadoras de deficiência. Constata-se, através da média, uma ênfase positiva na questão que se refere a gostar de si mesmo, o que pode estar associado a uma necessidade de fazer frente às discriminações sociais.

\begin{tabular}{ccccc}
\hline Item & carga & $\mathbf{h}^{\mathbf{2}}$ & $\mathbf{x}$ & Descrição \\
\hline 06 & 0,68 & 0,61 & 3,37 & Maior dificuldade para realizar os projetos desejados do que as pessoas \\
& & & & não portadoras de deficiência \\
07 & 0,86 & 0,82 & 3,31 & Dificuldade para se candidatar a emprego por ser PPD \\
\hline
\end{tabular}

Tabela 4. Matriz Fatorial do Fator Barreiras à Cidadania $($ Alfa $=0,79)$. Fonte: Dados da pesquisa, 2004.

Esse fator representa o construto que expressa a percepção da PPD sobre barreiras à cidadania, principalmente no que se refere às dificuldades em realizar seus projetos e às dificuldades em se candidatar a um emprego, o que dá ao portador de deficiência menores possibilidades de exercer sua cidadania em plenitude. Em relação à amostra pesquisada, as médias encontradas demonstram uma tendência à concordância quanto à presença dessas barreiras em seu cotidiano.

\section{c) Correlação dos fatores com Renda Familiar e Nível de Escolaridade}

A Tabela 5 mostra a relação entre os fatores e as variáveis demográficas renda familiar e nível de escolaridade.

\begin{tabular}{lccccc}
\hline Variáveis & F1 & F2 & F3 & $\begin{array}{c}\text { Renda } \\
\text { familiar }\end{array}$ & Escolaridade \\
\hline Renda familiar & - & - & $-0,348^{*}$ & - & $0,524^{* *}$ \\
Escolaridade & - & - & $-0,438^{* *}$ & $0,524^{* *}$ & - \\
\hline * Correlação é significante ao nível de 0,05 (2-tailed) & & & \\
** Correlação é significante ao nível de 0,01 (2-tailed) & & \\
F1 = Percepção das Relações Sociais e Aprendizagem & & \\
F2 = Auto-Estima & & & \\
F3 = Barreiras à Cidadania & & & \\
\hline
\end{tabular}

Tabela 5. Correlação de Pearson entre os fatores de Inserção Social e no Mercado de Trabalho e Variáveis Demográficas. Fonte: Dados da pesquisa, 2004. 
Observa-se na Tabela 5 que:

1) Quanto menor a renda familiar, maior as barreiras ao exercício da cidadania percebida pelas PPDs;

2) Quanto menor a escolaridade, maior as dificuldades percebidas para a realização dos projetos pessoais e a dificuldade em se candidatar a um emprego (fator barreiras à cidadania); e

3) Quanto maior a renda familiar, maior a escolaridade das PPDs.

\section{Considerações Finais}

Tendo como referência o conjunto de dados dessa pesquisa, foi possível constatar que as PPDs em Cabo Verde possuem dificuldade para ingressar no mercado formal de trabalho e que não existe, no país, a legalização do direito ao trabalho para essas pessoas.

O percentual de pessoas que não trabalham ou que estão no mercado informal de trabalho $(81,3 \%)$ é um indicador indiscutível da dificuldade das PPDs em se inserirem no mercado formal de trabalho. Além disso, a análise das questões que apresentaram um alto índice de não resposta indica o desconhecimento das pessoas em relação às condições de trabalho e à falta de experiência anterior no mercado formal.

Outras questões com grande número de não respostas foram as relativas aos direitos das PPDs, o que reflete a ausência de políticas públicas efetivas em relação a essa população e uma falta de divulgação de direitos previstos na legislação do país.

Analisando os itens do questionário, foram encontrados três fatores principais no que se refere à inserção social e no mercado formal de trabalho: percepção das relações sociais e aprendizagem; auto-estima e barreiras à cidadania.

O fator percepção das relações sociais e aprendizagem identifica a importância do papel da família e da sociedade na concepção que a própria PPD tem de sua capacidade de aprendizagem no espaço escolar. Nesse sentido, existe uma interdependência entre a percepção de falta de confiança da família nas possibilidades das PPDs, o tratamento mais ou menos preconceituoso percebido por parte da sociedade e o sentimento das PPDs em relação às suas próprias capacidades. O que se depreende dos resultados encontrados é que as pessoas que fizeram parte da pesquisa, em sua maioria, sentiram-se tratadas com preconceito e não tiveram a confiança necessária da família, já que aproximadamente $60 \%$ das pessoas possuíam apenas o primário completo. No entanto, é preciso considerar que a baixa escolaridade também está associada positivamente com a baixa renda familiar dessas 
pessoas, o que pode ter contribuído objetivamente para dificultar o acesso à educação de muitas PPDs.

Quanto ao fator relacionado à auto-estima, constata-se que as pessoas têm um sentimento positivo em relação a si mesmas. No entanto, esse sentimento é interdependente da forma como as PPDs acham que são percebidas pela sociedade. Na amostra pesquisada, apesar de haver uma ligeira tendência das PPDs em concordarem que são consideradas inferiores às pessoas não portadoras de deficiência, há uma resposta enfática em relação ao gostar de si mesmas. Essa tendência de resposta pode indicar uma reação defensiva frente às discriminações sociais e uma busca por continuar a existir, apesar das dificuldades. A configuração desse fator carece de novas pesquisas, para a ampliação de suas repercussões para as PPDs.

O fator barreiras à cidadania identifica duas grandes dificuldades das PPDs, as quais podem comprometer o pleno exercício da cidadania por parte delas. As duas dificuldades dizem respeito à realização de seus projetos pessoais e à possibilidade de se candidatar a um emprego. Essas dificuldades denunciam as desigualdades, reiteradas pela sociedade que nega uma oportunidade igual para todos, além de tornar mais custosa a possibilidade de independência e de autonomia por parte das PPDs. A percepção dessas dificuldades das PPDs é ratificada pelo número de PPDs que estão fora do mercado formal de trabalho (80,3\%). Apesar disso, as percepções dessas barreiras são minimizadas com o aumento da renda familiar e da escolaridade das PPDs, reafirmando a assertiva de Bourdieu $(1996,1998)$ a de que o capital econômico e o capital cultural são os princípios de diferenciação por excelência na sociedade atual. Além disso, a presença de capital econômico e cultural aumenta as possibilidades de estabelecimento de redes de relações por parte dessas pessoas (capital social), facilitando a inserção social e no mercado formal de trabalho.

Os resultados da pesquisa também reafirmam a correlação positiva entre renda familiar e escolaridade, o que confere uma posição privilegiada às PPDs que possuem esses dois tipos de capital, dando-lhes, inclusive, condições de fazerem com que a sociedade se sensibilize em relação às dificuldades e às potencialidades das PPDs.

Esses resultados da pesquisa indicam a necessidade do país em repensar as políticas públicas destinadas às PPDs, ampliando as discussões sobre a questão da igualdade social. Por outro lado, também existe a necessidade de conscientização dos gestores das empresas, em relação à importância da diversidade nas organizações, uma vez que a abertura ao 
mercado externo, por parte das empresas caboverdianas, implica o realinhamento de políticas de gestão, visando refletir a responsabilidade social das empresas.

\section{Referências bibliográficas}

BATAVIA, A. I.; SCHRINER, K. (2001). The Americans with Disabilities Act as engine of social change: models of disability and the potencial of civil rights approach. Policy Studies Journal, v. 29, n. 4, p. 690-702.

BATISTA, C. A. M. (2004). Inclusão: construção na diversidade. Belo Horizonte: Armazém de Idéias. 188 p.

BOURDIEU, P. (1996). Razões práticas: sobre a teoria da ação. Campinas: Papirus. 224 p. (1998). O poder simbólico. 2. ed. Rio de Janeiro: Bertrand Brasil. 314 p.

BOURDIEU, P.; WACQUANT, L. (1992). An invitation to reflexive sociology. Chicago: The University of Chicago Press. 319 p.

CABO VERDE. Código Laboral (2001). Documento da Câmara Muncipal da Ilha do Sal de Cabo Verde sobre medidas da legislação laboral do país, 25 de julho de 2001.

FRANÇA, J. L.; VASCONCELOS, A. C. (2004). Manual para normalização de publicações técnico-científicas. 7. ed. Belo Horizonte: UFMG. 242 p.

FREITAS, M. N. C.; MARQUES, A. L.; SCHERER, F. L. (2004). Inclusão no Mercado de Trabalho: um estudo com Pessoas Portadoras de Deficiência. In: ENCONTRO ANUAL DA ASSOCIAÇÃO NACIONAL DE PÓS-GRADUAÇÃO E PESQUISA EM ADMINISTRAÇÃO, 28., 2004, Curitiba. Anais eletrônicos... Curitiba: EnANPAD. CDROM.

GOFFMAN, E. (1982). Estigma: notas sobre a manipulação da identidade deteriorada. 4. ed. São Paulo: J. Zahar. 158 p.

GOSS, D.; GOSS, F.; ADAM-SMITH, D. (2000). Disability and employment: a comparative critique of UK legislation. The International Journal of Human Resource Management, 11:4, p. 807-821, Aug.

INSTITUTO NACIONAL DE ESTATÍSTICA (2000). Cabo Verde em números: Censo 2000. Disponível em: <http://www.ine.cv>. Acesso em: 11 jan. 2005.

LEWIS, G. B.; ALLEE, C. L. (1992). The impact of disabilities on Federal Career Success. Public Administration Review, v. 53, n. 4, p. 389-397, July/Aug.

MALHOtRA, N. K. (2001). Pesquisa de marketing: uma orientação aplicada. 3. ed. Porto Alegre: Bookman. 720 p.

MARTINS, S. M. F. C. (1996). Deficiência física e mercado de trabalho: o ponto de vista do trabalhador deficiente. Consciência, Palmar-Pr, v. 10, n.1, p. 55-70, jan./jun. 
MINISTÉRIO DAS RELAÇÕES EXTERIORES DO BRASIL (2005). Política externa. Informações sobre Cabo Verde, 2005. Disponível em: <www.mre.gov.br/portugues/politicaexterna>. Acesso em: 20 jan. 2005.

OLIVEIRA, M. H. A. (1993). Núcleos cooperativos: uma perspectiva profissional para o portador de deficiência. Em Aberto, Brasília, ano 13, n. 60, p. 106-108, out./dez.

SILVA, M. C. O. (1993). Uma questão de competência. São Paulo: Memnon. 127 p.

SCHUR, L (2002). The difference a job makes: tehe effects of employment among people with disabilities. Journal of Economic Issues, v. 36, issue 2, p. 339-348, Jun.

\section{Dados dos Autores}

\section{Maria Nivalda de Carvalho-Freitas}

Doutoranda em Administração pelo Centro de Pós-Graduação e Pesquisas em Administração (CEPEAD), da Faculdade de Ciências Ecônomicas (FACE), pertencente à Universidade Federal de Minas Gerais (UFMG). Mestre e Psicóloga do Trabalho. Professora do Departamento de Psicologia da Universidade Federal de São João del-Rei (UFSJ).

Telefones: (32) 3371-1117 - (32) 9981-3302

Endereço Profissional: Praça Dom Helvécio, 74 - Bairro Fábricas, São João del-Rei - MG - 36301-160

Endereço Residencial: Rua Felipe Marchetti, 80 - Bairro Vila Marchetti, São João del-Rei - MG - 36307-248

carvalhofreitas@mgconecta.com.br

\section{Nadine Helene Silva Rocha}

Aluna do Curso de Engenharia Elétrica da Universidade Federal de São João del-Rei (UFSJ). Natural de Cabo Verde - África.

\section{Raquel Margarida Nascimento Lopes}

Psicóloga, graduada pela Universidade Federal de São João del-Rei (UFSJ). Natural de Cabo Verde - África.

\section{Sónia Helena Lima Silva}

Aluna do Curso de Psicologia da Universidade Federal de São João del-Rei (UFSJ). Natural de Cabo Verde - África.

\section{Walter Leany Baptista Spínola}

Psicólogo, graduado pela Universidade Federal de São João del-Rei (UFSJ). Natural de Cabo Verde - África.

\section{Como citar este artigo}

CARVALHO-FREITAS Maria Nivalda de et al. Inclusão social de pessoas com deficiência em Cabo Verde - África. Revista Moçambras: acolhendo a alfabetização nos países de língua portuguesa, São Paulo, ano 1, n. 2, 2007. Disponível em: <http://www.mocambras.org>. Publicado em: março 2007. 